\title{
In vivo Evaluation of Mucociliary Clearance in Mice
}

Kyle S. Feldman ${ }^{1}$, Maliha Zahid ${ }^{1}$

${ }^{1}$ Department of Developmental Biology, University of Pittsburgh School of Medicine

\section{Corresponding Author}

Maliha Zahid

maz7@pitt.edu

\section{Citation}

Feldman, K.S., Zahid, M. In vivo Evaluation of Mucociliary Clearance in Mice. J. Vis. Exp. (166), e61929, doi:10.3791/61929 (2020).

\section{Date Published}

December 18, 2020

DOI

$10.3791 / 61929$

URL

jove.com/video/61929

\section{Abstract}

Respiratory motile cilia, specialized organelles of the cell, line the apical surface of epithelial cells lining the respiratory tract. By beating in a metachronal, synchronal fashion, these multiple, motile, actin-based organelles generate a cephalad fluid flow clearing the respiratory tract of inhaled pollutants and pathogens. With increasing environmental pollution, novel viral pathogens and emerging multi-drug resistant bacteria, cilia generated mucociliary clearance (MCC) is essential for maintaining lung health. MCC is also depressed in multiple congenital disorders like primary ciliary dyskinesia, cystic fibrosis as well as acquired disorders like chronic obstructive pulmonary disease. All these disorders have established, in some case multiple, mouse models. In this publication, we detail a method using a small amount of radioactivity and dual-modality SPECT/CT imaging to accurately and reproducibly measure MCC in mice in vivo. The method allows for recovery of mice after imaging, making serial measurements possible, and testing potential therapeutics longitudinally over time. The data in wild-type mice demonstrates the reproducibility of the MCC measurement as long as adequate attention to detail is paid, and the protocol strictly adhered to.

\section{Introduction}

Cilia are microtubule based cellular organelles conserved across evolutionary history from algae to humans. They emanate from cell surfaces and have a number of functions ${ }^{1}$, ranging from recognition of local environmental sensory signals to motility, functions which can be traced back from humans to early unicellular eukaryotic organisms ${ }^{2,3}$. Cilia can be non-motile and single serving as a cell's specialized antenna to process environmental signals; or motile and multiple, beating in synchronized, metachronal waves to generate fluid flow, such as in the lining of the fallopian tubes and the upper and lower airways, except for the terminal bronchioles leading to the alveoli ${ }^{1,2}$.

The extensive epithelial surface of the respiratory tract is exposed to a constant barrage of contamination in the form of a variety of potentially hazardous inhaled pollutants and pathogens, necessitating a defense. One key defense mechanism is the mucociliary apparatus of the tracheobronchial tree, where a continuous flow of secreted 
mucus is mechanically transported out of the airway by the beating of multiple motile cilia lining the apical surfaces of the tracheo-bronchial epithelial cells. These function to entrap inhaled contaminants, and through their continuous, synchronal beating, transport them cephalad ${ }^{4,5}$.

Cilia have been demonstrated to have key roles such as in the development of left-right patterning in developing embryos, where motile cilia at the embryonic node break symmetry $^{6}$. Mutations in cilia related genes have been linked to diseases such as congenital heart disease (CHD) due to the asymmetric structure of the heart ${ }^{6}$. Recent studies have reported a high incidence of ciliary dysfunction in the respiratory tracts of patients with $\mathrm{CHD}$, as well as an increased prevalence of post-operative respiratory complications and chronic respiratory tract symptoms in the upper and lower airways $7,8,9,10$. Patients with CHD and ciliary dysfunction, with or without heterotaxy, have been demonstrated to have increased risk of respiratory complications and negative respiratory outcomes postoperatively $5,8,10$. Beyond their roles in signaling and development, the importance of airway cilia has been demonstrated by ciliopathies, of which a prime example is primary ciliary dyskinesia (PCD). PCD is a congenital disorder resulting from a number of mutations affecting the motile respiratory cilia, leading to recurrent lung infections, bronchiectasis, and potentially the need for lung transplantation $^{11}$. Additionally, even though cilia are normal in cystic fibrosis (CF), most common congenital disorder in the Caucasian population, MCC is impaired due to thick, viscous mucus resulting from mutations in the CFTR gene ${ }^{12}$. There are multiple mouse models of PCD and CF, as well as an ever-increasing number of models of CHD. Ultimately cilia are versatile structures with many key roles, and a method to assess the function of motile respiratory cilia in vivo can be valuable for pre-clinical study, and assessing effects of mutations as well as drugs on mucociliary clearance $(\mathrm{MCC})^{13}$. The method would also be valuable in assessing effects of novel drugs, gene therapy or interventions on MCC in these mouse models.

There are many different models that have been used to assess MCC. One notable method involves the use of methylene blue dye that has been instilled into the bronchus, with clearance measured by fiberoptic measurement of dye movement ${ }^{14}$. This method is limited by the ability to observe the movement of the dye, which is more routine in humans than in pre-clinical mouse models. Another notable method is synchrotron phase-contrast $\mathrm{X}$-ray imaging $(\mathrm{PCXI})$, which can be used to track individual particles in an airway. This method is relatively new and not widely accessible ${ }^{15}$. There are numerous ex vivo methods of assessing the airway by excising a trachea for video-microscopy, however these models provide little utility in human patients ${ }^{16}$. High resolution techniques for cilia imaging such as optical coherence tomography are limited in the same way ${ }^{17}$.

In this article, we present a reproducible method to measure MCC in vivo that has been used to measure lung clearances in myriad animal models, as well as study MCC in chronic obstructive pulmonary disease and assess the effects of immunosuppressive drugs ${ }^{18,19}$. This method tracks the clearance of the radiopharmaceutical $99 m_{\text {technetium-sulfur }}$ colloid ( $\left.{ }^{99 m} \mathrm{Tc}-\mathrm{Sc}\right)$, an insoluble particulate radiotracer, after instillation into the lungs. The radionuclide can then be tracked using single photon emission computed tomography $(\mathrm{SPECT})^{18,20}$. We have further refined this technique for measuring MCC by using dual modality SPECT and computed tomography (CT) imaging with co-localization of radioisotope counts to the lungs and measuring the decrease 
in these counts over 6 hours. Dual-modality imaging, with co-registration of CT and SPECT images allows for accurate localization of radiation counts to our region of interest, the lungs. Although we describe in detail the method for MCC measurement in mice, the protocol can be adjusted to study MCC in rats. The collimators would need to be adjusted as well as radiation dose. In our view, mouse MCC scans are more technically challenging due to the small animal size, but more useful than rats due to the large number of established mouse models of a number of human disorders. Additionally, due to their lower cost and cost of maintenance in animal colonies, a larger sample size is more feasible in mice.

\section{Protocol}

The University of Pittsburgh's Institutional Animal Care and Use Committee approved all animal protocols specified in this publication prior to undertaking any of these animal experiments.

NOTE: This protocol details how to perform in vivo mucociliary clearance studies utilizing radionuclide imaging with a dualmodality SPECT/CT scanner. The techniques demonstrated are running system calibrations, anesthetizing mice, tracheal intubation of mice, instilling the isotope into the lungs, dual-modality imaging, co-registration of these images, and analysis.

\section{SPECT/CT system setup}

1. Design an appropriate workflow and set up prior to running experiments using living animals.

1. Use a SPECT acquisition consisting of 60 projections with a step size of $6^{\circ}$ between projections with a $40 \mathrm{~cm}$ radius of rotation. The CT acquisition consists of 220 projections with a $1.6^{\circ}$ angle between projections.

2. Ensure that the system has the correct MWB collimators for mice and SPECT imaging in place. If the inappropriate collimators are installed, use the collimator wizard to install the correct ones.

3. Run the necessary system calibrations to prepare the system for use.

NOTE: The SPECT and CT components of the scanner need calibration. Calibrate the CT components using a source conditioning and a Dark/Light (D/L) calibration once a day, a Center Offset (COS) calibration every 2 weeks, and evaluate the x-ray hardware every month. The SPECT components need to be calibrated once a year.

1. To evaluate the x-ray hardware, check the Evaluate X-ray hardware box during CT calibrations (Supplemental CT Calibration Menu).

2. To perform source conditioning, check the Perform source conditioning box during CT calibrations (Supplemental CT Calibration Menu).

3. To perform a $\mathrm{D} / \mathrm{L}$ calibration, check the $\mathrm{D} / \mathrm{L}$ box next to the CT acquisition protocol used during experiments during CT calibrations. Uncheck all other protocols (Supplemental CT Calibration Menu).

4. To perform a COS calibration, replace the bed with the calibration ring tool, adjust the bed type settings to match in the motion control settings, and check the COS box next to the CT acquisition protocol used during experiments during CT calibrations. Uncheck all other protocols (Supplemental CT Calibration Menu, Supplemental Calibration Ring). 


\section{Mouse Intubation and Instillation}

1. Weigh the mice to be scanned. If scanning multiple mice, take care to mark the mice for identification purposes using methods such as ear punching or marking of the tail.

2. Anesthetize a mouse using $1.5 \%$ isoflurane with a gas flow of $2 \mathrm{~L} / \mathrm{min} \mathrm{O}_{2}$ in a gas chamber for $\sim 5$ minutes to produce anesthesia of sufficient depth, until breathing slows to $\sim 55-65$ breaths per minute ${ }^{16}$ (Figure 1A).

3. Remove the mouse from the chamber and suspend by the front incisors on an intubation stand at a $45^{\circ}$ incline. Equip the intubation stand with a nose cone to ensure the mouse is anesthetized during the intubation (Figure 1B).

4. Connect one end of a $50 \mu \mathrm{m}$ fiber optic wire to a light source and thread a 20-gauge cannula over it using the wire to act as a guide (Figure 1C).

5. Open the mouth of the mouse and pull the tongue forward using blunt forceps. Illuminate the guide wire and use it to visualize the vocal cords (Figure 1D).

6. Pass the guide wire through the vocal cords so that the wire is just beyond the vocal cords and resting in the upper trachea. Slide the 1 inch cannula forward along the wire to intubate the mouse, passing the cannula deep enough so that the hub of it is against the animal's incisors (Figure 1E). Remove the wire leaving the cannula in place.

7. Test the intubation by briefly plugging the cannula with a finger and checking for changes in breathing. Halted breathing or strained breathing while plugging and accelerated breathing upon release are signs of proper tracheal intubation. If there is no change in respiratory patterns upon plugging the cannula, the latter is likely in the esophagus.

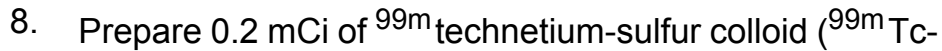
Sc) in a volume of $10 \mu \mathrm{L}$, and pipette into the cannula. Allow the mouse to spontaneously inhale it into the lungs over 1-2 min (Figure 1F). Remove the cannula before transferring the mouse to the pallet of the scanner.

NOTE: The radionuclide was prepared and filtered by Cardinal Health.

\section{SPECT/CT Imaging}

1. Transfer the mouse to a $25 \mathrm{~mm}$ pallet with a nose cone and secure with tape, taking care to not tape the chest and abdomen too tightly to avoid impairing respiration. Take care to remove any metal ear tags attached to the mouse.

2. Prepare a radioactive phantom consisting of $0.05 \mathrm{mCi}$ in $200 \mu \mathrm{L}$ and place this amount in a $0.2 \mathrm{~mL}$ PCR tube. Position the tube by taping to the pallet under the lower abdomen of the mouse, avoiding overlap with the lungs. NOTE: The phantom is used for the purpose of coregistering CT and SPECT images, as well as a negative control for clearance.

3. Insert the mouse into the SPECT/CT system, select the imaging workflow, and run Setup.

4. Set up the positioning of the detectors on the mouse, and run the imaging workflow.

5. Prepare a cage for mice that have received radioactivity post-procedure, with unrestricted access to food and water, and clear labeling using a radiation safety sticker.

6. Upon completion of the workflow, remove the mouse from the imaging pallet, and allow it to recover in the prepared cage for a duration of 6 hours between 
scans (end of scan 1 to beginning of scan 2) with ad libitum access to food and water. $6 \mathrm{~h}$ was chosen as it corresponds to the time period in which linear clearance depending on cilia function is taking place with very little alveolar clearance.

7. After 6 hours, re-anaesthetize the mouse and scan, along with the phantom, using the same workflow to measure the amount of isotope cleared from the airways.

NOTE: It is critical to allow the mouse to recover as uninterrupted anesthesia with isoflurane for 6 hours will lead to a significant cilia-depressant effect, resulting in near zero mucociliary clearances.

\section{Analysis}

1. After imaging, perform post-processing to reconstruct complete 3D stack images.

1. Histogram the SPECT images using the factory standard settings for ${ }^{99 \mathrm{~m}} \mathrm{Tc}$, and then reconstruct using a MAP3D algorithm and point spread function (PSF) reconstruction.

NOTE: The reconstruction was performed using 8 iterations and 6 subsets. An effective reconstruction needs a ratio of subsets to projections at $1: 10$ or divide evenly into the number of projections, so 6 subsets were used due to the acquisition using 60 projections.

2. Reconstruct the CT images using the Feldkamp algorithm and a Shepp-Logan filter.

NOTE: The reconstruction was performed using 4 iterations.

2. Process the CT and SPECT images in FIJI ImageJ ${ }^{21}$ using the reslice tool to generate coronal view images from the default axial images. Then perform a z-stack sum projection on the SPECT image to add the count data from each slice and generate a single image for ease of analysis.

3. Resize and co-register the CT and SPECT images using the phantom Eppendorf tube as a reference (Figure 2A,B). Track and use consistent resize measurements across all samples.

4. Binarize the CT image using auto thresholding, followed by inverting the stack, and performing a z-stack sum projection to generate an outline of the lungs for analysis (Figure 2C).

5. Rotate the CT and SPECT images and merge the image using the channel tools. Calculate MCC by drawing an ROI around the right lung and measuring (Figure 2D).

NOTE: This measurement will be of the total counts in the right lung for the 0 and 6 hour time points, with the 6 hour images corrected for radioactive decay using the formula: $N(t)=N_{0} e^{-t} \cdot{ }^{99 m}$ Tc-Sc has a decay constant of $3.21 e^{-5}$ per second with a half-life of $\sim 6$ hours. These values can then be used to calculate a percent clearance. NOTE: Right lung is chosen for ROI drawing and measuring counts as the mucociliary clearance will transport the radioisotope out of the lungs to the pharynx from where it will be swallowed and end up in the stomach. Quite frequently, counts can be seen in the stomach that may overlap with the left lung and hence produce erroneous counts. This confounding can be avoided by measuring counts in the right lung only.

\section{Representative Results}

Using this protocol, we anesthetized mice in an isoflurane chamber (Figure 1A). After achieving an adequate level of anesthesia, mice were placed onto vertical supports 
(Figure 1B) and the vocal cords were visualized using an illuminated guide wire (Figure 1C-1D). The mice were intubated and instilled with $0.2 \mathrm{mCi}^{99 \mathrm{~m}} \mathrm{Tc}-\mathrm{Sc}$ in volumes of $10 \mu \mathrm{L}$ through a cannula and mice allowed to spontaneously inhale into the lungs (Figure 1E-1F). After image acquisition and processing, the CT and SPECT images were colocalized (Figure 2A) using the phantom tube as a landmark (Figure 2B). Masks of the lungs were generated from the CT image (Figure 2C) and used to draw ROls around the right lung for analysis at 0 (Figure 2D) and 6 hours (Figure
2E-2F). To test reproducibility of the protocol, a total of 8 mice were scanned twice on different days with identical experimental conditions, with analysis using a paired ttest showing no significant difference between the repeat scans ( $p$-value=0.9904) (Figure 3A). An additional 2 mice were scanned three times on different days with identical experimental conditions, with analysis using one-way ANOVA showing significant matching between the repeat scans $(p-$ value of 0.0041 ) (Figure 3B). A total of 8 mice were scanned and two representative images were displayed (Figure 4).

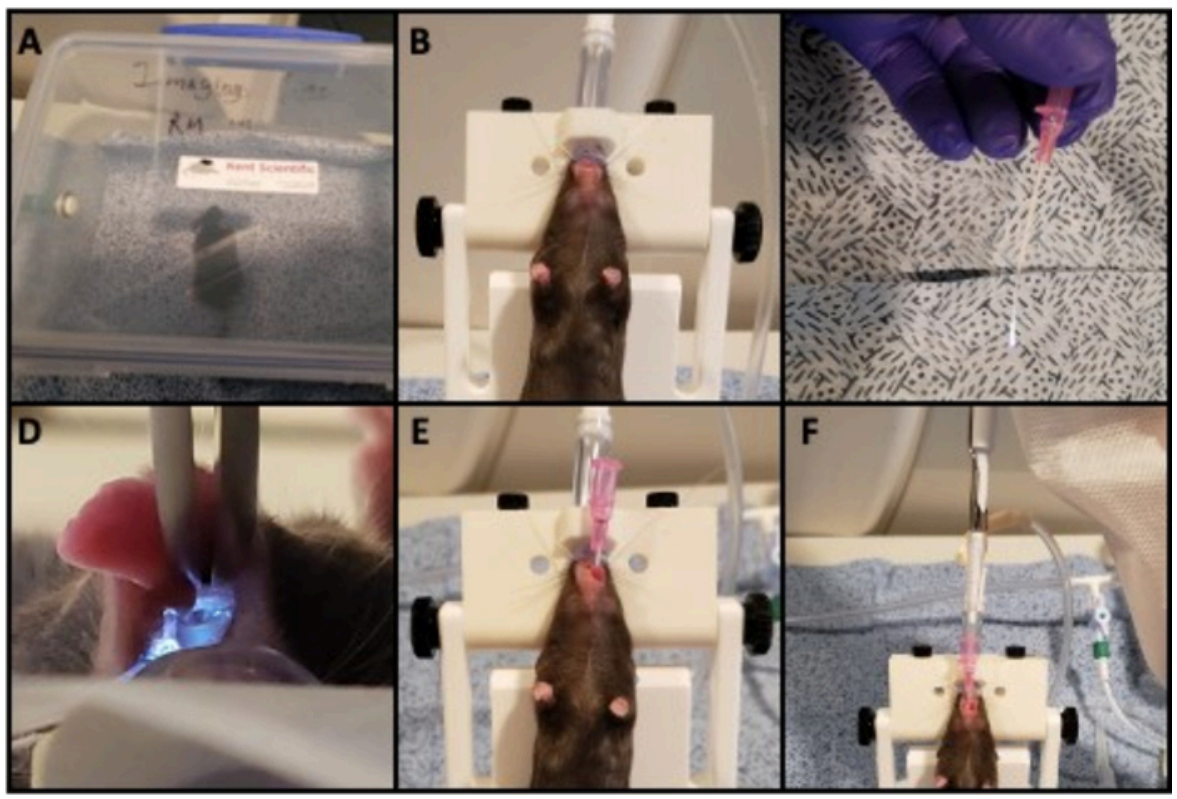

Figure 1: Mouse intubation and isotope instillation. Images of the steps needed to intubate and instill isotope into the airway. A) The mouse is anesthetized in a chamber. B) The anesthetized mouse is placed onto a vertical support, suspended by the front incisors. C) An illuminated $0.5 \mathrm{~mm}$ fiber optic wire serving as a guide wire is prepared by running it through a $20 \mathrm{G}$ cannula. D) The mouth of the mouse is opened using forceps and illuminated using the lighted guidewire to visualize the vocal cords. E) The cannula is pushed across the vocal cords and guidewire is removed. F) Soluble isotope is instilled into the cannula using a pipette and the mouse allowed to spontaneously inhale the isotope into the lungs. Please click here to view a larger version of this figure. 


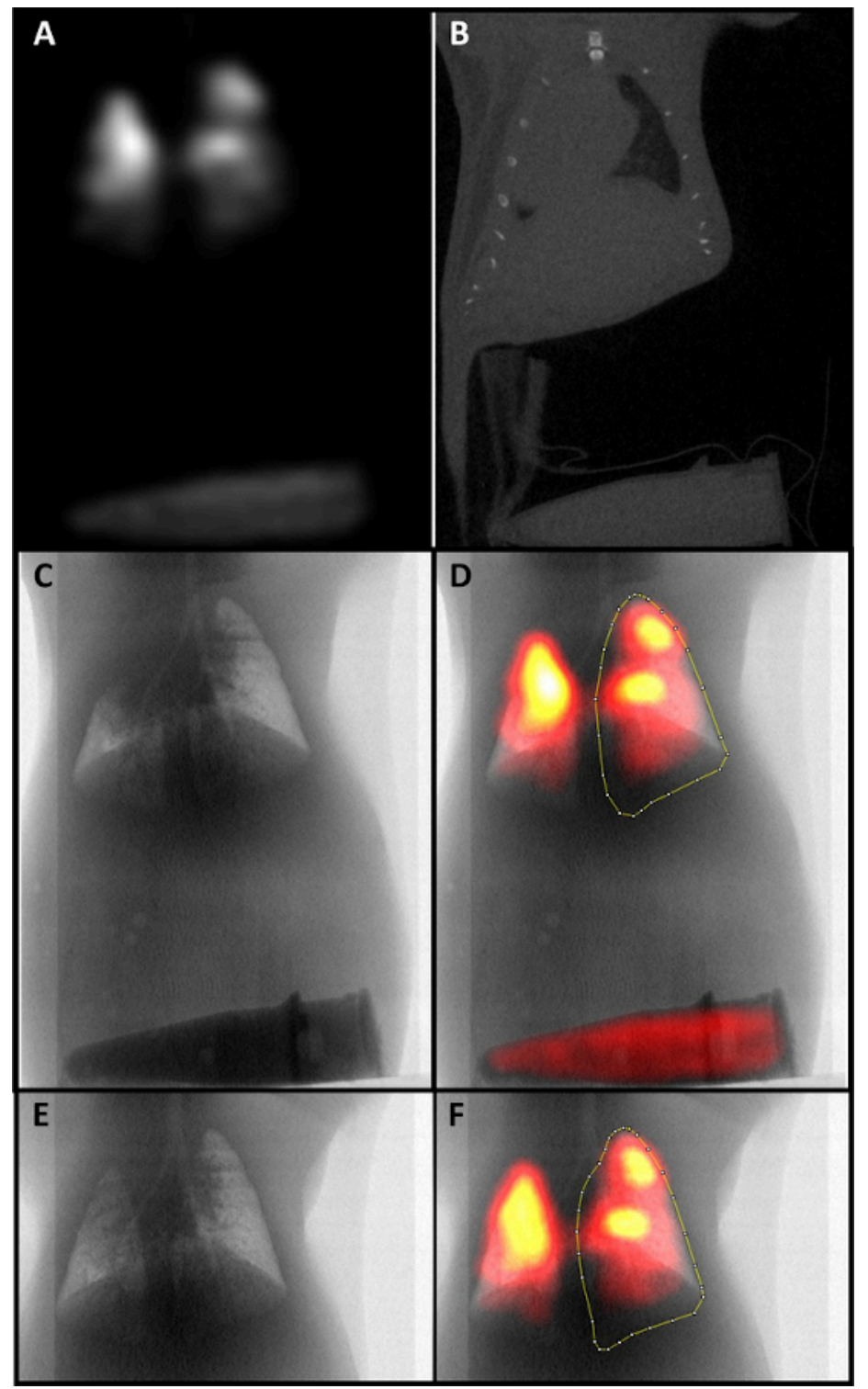

Figure 2: SPECT/CT images of an MCC scan. A) A SPECT image that has been co-localized with a CT image. B) A CT image with a visible phantom tube that was used for co-localization. C) A mask of the airway derived by binarizing the CT image and performing a z-stack sum projection. D) The CT mask co-localized with the SPECT image. An ROI for analysis has been drawn around the right lung. E) A mask of the airway at 6 hours. F) A CT and SPECT co-localized image of the airway at 6 hours with an ROI for analysis. Please click here to view a larger version of this figure. 


\section{A}

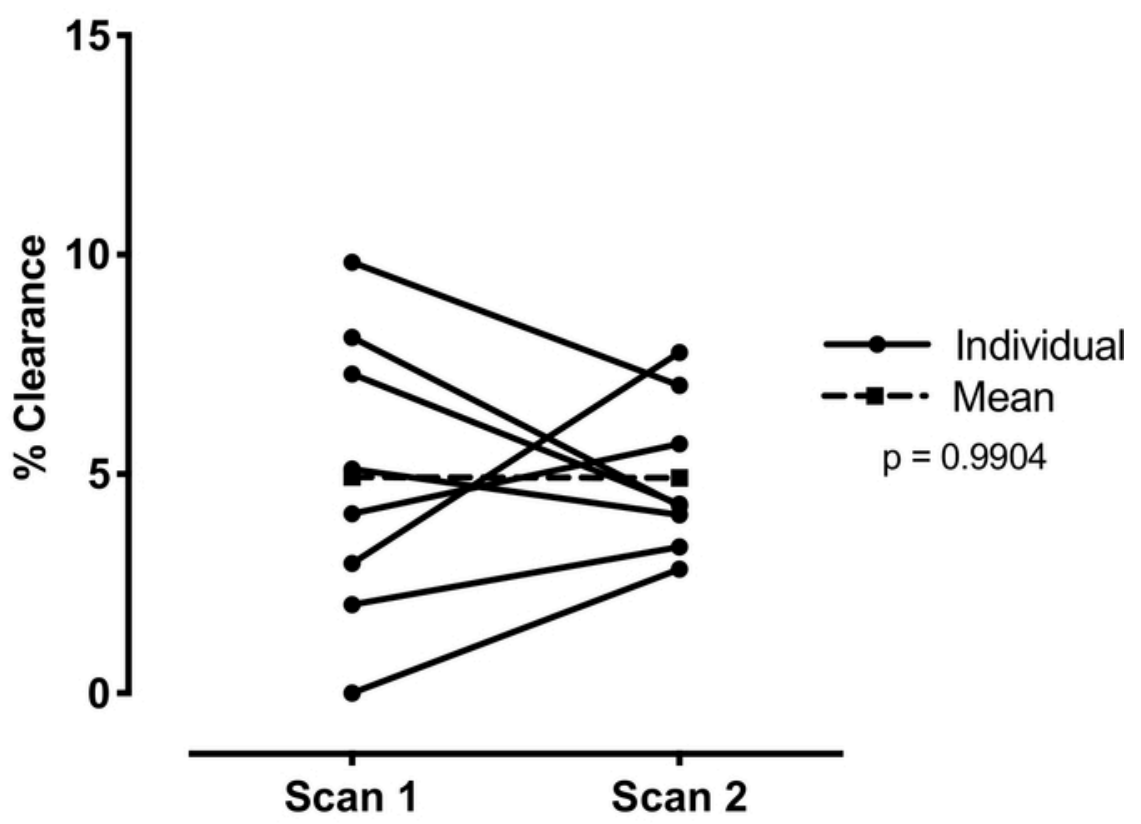

B

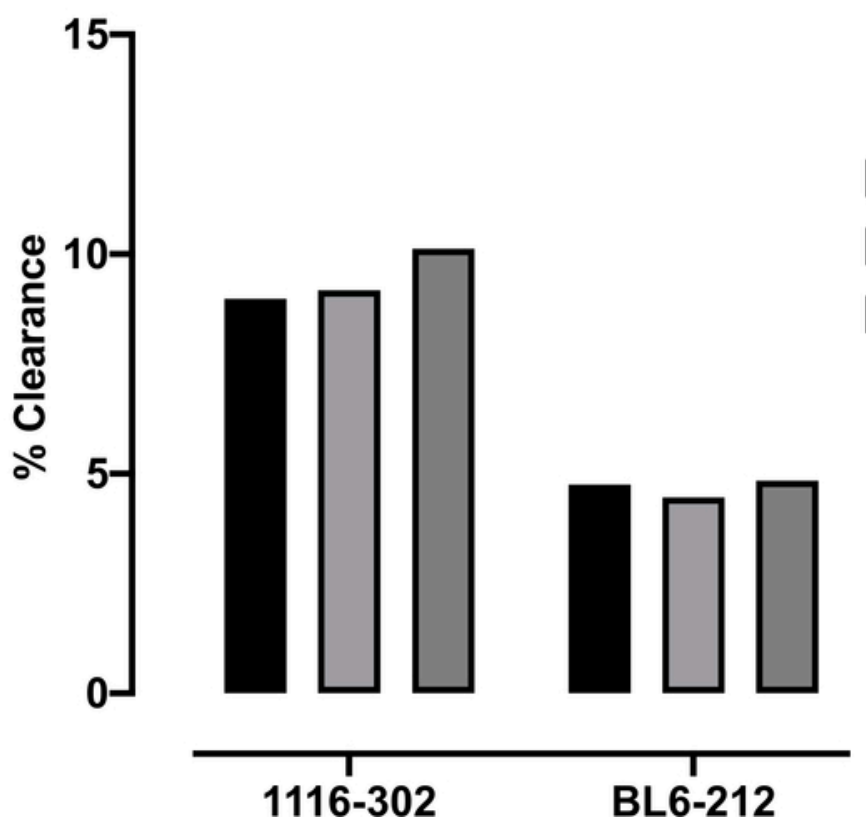

Figure 3: Clearance measurements of the same mice across multiple scans. A) Two individual repeat clearance were measured for 8 mice with no changes in experimental conditions. A paired t test showed that there was no significant difference between the repeat scans with a p-value of 0.9904. B) Three individual repeat clearances were measured for two mice with no changes in experimental conditions. A one-way ANOVA showed there was significant matching between the repeat scans with a $p$-value of 0.0041 . Please click here to view a larger version of this figure. 


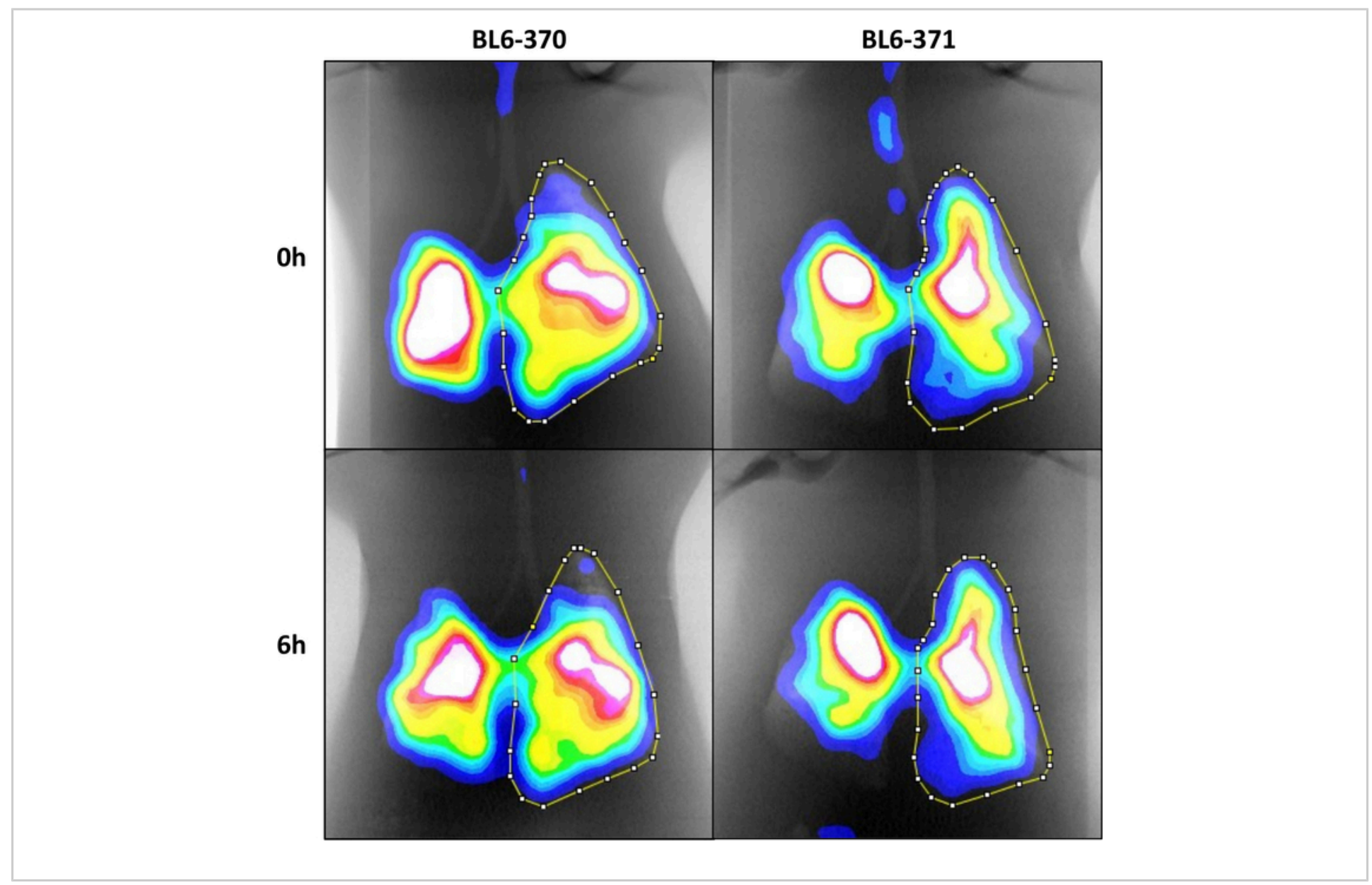

Figure 4: Co-localized SPECT/CT images of the 0 and 6 hour airway in 2 mice with ROls drawn at 0 and 6 hours outlining the right lung. Please click here to view a larger version of this figure.

\section{Supplemental Figure 1: A video of the vocal chords} illuminated by a fiber optic wire with the effect of breathing visualized. Please click here to download this figure.

Supplemental Files. Please click here to download these files.

\section{Discussion}

The role of motile respiratory cilia in both disease and development continues to evolve and be better appreciated. Synchronous, metachronal beating of multiple motile cilia on the apical surface of cells lining the tracheobronchial tree generate cephalad flow producing mucociliary clearance or MCC. MCC is compromised in ciliopathies like $P C D^{22}$, acquired diseases like $\operatorname{COPD}^{18}$, and its importance is being recognized in CHDs, not traditionally considered to be ciliopathies. Recent data has shown respiratory ciliary dysfunction in both $\mathrm{CHD}$ with heterotaxy ${ }^{23}$ and without heterotaxy ${ }^{7}$. Such motile cilia dysfunction was shown to translate into greater respiratory symptoms ${ }^{9}$ as well as greater post-operative morbidity ${ }^{8}$. Most, if not all, of these diseases, have mouse models available and our protocol for 
measuring MCC in mice is a valuable tool that can be utilized to test potential therapeutics.

Animal models provide utility for understanding diseases and the development of therapies. In vivo animal imaging provides further utility with the ability to acquire multiple data points from the same animals, without the need to sacrifice the animals, allowing investigators to follow longitudinal course of disease as well as study duration of treatment effects. The mouse model of MCC has been developed over the course of decades by multiple investigators, initially being performed on beagle dogs using planar scintigraphy, a two dimensional nuclear imaging technique ${ }^{24}$. The technique was adapted for use in mice a decade later, followed by adaptation to SPECT imaging a decade after that ${ }^{25,26}$. The development of this technique in mouse models was a major development in the relevance of this technique, due to the availability of multiple mouse models of human diseases like PCD in which ciliary function is significantly altered. MCC has been assessed in mouse models of lung denervation and immunosuppression, and has the potential to be used in conjunction with other models ${ }^{19,26}$. MCC measurement studies in human patients with airway diseases such as CF, asthma, PCD, and ciliopathies associated with CHD have been conducted, and have yielded results that the technique can aid both studies of lung physiology and therapeutic efficacy ${ }^{13}$.

An important part of this protocol is setting up acquisitions with the correct imaging parameters to acquire accurate images for quantification. A number of factors are key when designing SPECT acquisition settings, including which collimators are used, the number of projections to acquire per revolution, and rotation step size. Collimator selection is a major factor in the sensitivity and resolution of the acquisition, and acquisition settings may need to be tailored to the collimator being used ${ }^{27}$. Alternately, when using bigger animals like rats, the collimators would need to be adjusted. Multiple pinhole collimators for example are more sensitive, but care should be taken when selecting a step size in order to avoid overlapping projections and causing undesired multiplexing, which can further increase sensitivity of the acquisition at the expense of some image ambiguity that can cause reconstruction artifacts ${ }^{25}$. Reconstruction setup is also key to generate quantifiable images. MAP3D is a commonly used iterative reconstruction algorithm, and PSF is a common reconstruction model. Both are reliable for reconstructing images, but care should be taken when setting the number of iterations and subsets. A higher number of iterations will increase the computational time required for the reconstruction, and increase the quality of the reconstruction with diminishing returns upon further increase.

In order to quantify images in ImageJ, the ideal measurement tool to use is RawlntDen, which outputs the sum value of pixels in a selection. When quantifying SPECT data across differently sized lung ROIs, the use of RawlntDen provides an absolute measure of counts and avoids adjusting the measurement to the area of the ROI, like the mean measurement would ${ }^{21}$.

This technique has a number of associated sources of error that the investigator should be cognizant of when applying this technique. A notable confounder is the use of anesthetic agents. Isoflurane is a fast acting, inhaled anesthetic that the mice recover from rapidly after completion of an acquisition. However, care should be taken to provide the mice with ample time to recover in their cages, and not kept anesthetized any longer than necessary. In our personal experience (unpublished data) mice that were kept 
anesthetized continuously using inhaled isoflurane between the 0 and 6 hour time-point showed negligible clearance. Likewise, a controlled dose of anesthetic is also necessary to ensure rapid recovery. When securing the animal to the pallet for imaging, the phantom tube used for co-registration should be kept low on the stomach to avoid artifacts from overlapping with the lungs. Likewise, to ensure a quality CT image, take care to remove any metal tags from the mouse to avoid artifacts from $\mathrm{x}$-ray scattering.

The current MCC protocol can be applied to myriad animal models. This technique has a negligible effect on the health of the animal scanned, is well tolerated by mice, and because of this it can be used with disease models without risking the health of already delicate mice. The strength of this methodology comes from it being an in vivo technique, which allows for the acquisition of consistent and repeatable measurements of airway function without the sacrifice of animals to excise tracheas for video-microscopy, that ex vivo models require ${ }^{26}$. The consistency of this technique in producing repeatable measurements across multiple scans of the same animals, allows for the same animal to be treated with different agents or potential therapeutics, and statistical comparisons made between the same animal to reduce biological variability inherent in any animal model, thereby reducing the sample size needed to show statistically significant differences.

The assessment of airway function using the MCC technique can be adjusted to a variety of animal models and applied to many different models of airway health, as well as testing new therapies. The airways of mouse models of PCD can be assessed using this technique, as well as models of COPD. Our method can also be utilized to study differential effects of various anesthetics on MCC that are in common clinical use. Finally, the effects of therapeutic agents on the airway can also be assessed using this model. As previously stated but bears repetition, as it is an in vivo measurement it allows for repeat MCC assessments over the course of a disease, as well as test benefits of therapeutic interventions over time. Additionally, mice are the most common laboratory animals used to mimic/study human diseases, with, in some cases, multiple transgenic mouse models of human disease available to choose from.

\section{Disclosures}

None related to this work.

\section{Acknowledgments}

M.Z. and K.S.F. and this work was supported by a grant awarded under the Pitt Innovation Challenge (PInCh), through the Clinical and Translational Science Institute at the University of Pittsburgh, and NHLBI R01 grant HL153407, awarded to M.Z.

\section{References}

1. Afzelius, B. A. Cilia-related diseases. Journal of Pathology. 204 (4), 470-477 (2004).

2. Mitchell, D. R. The evolution of eukaryotic cilia and flagella as motile and sensory organelles. Advances in Experimental Medicine and Biology. 607, 130-140 (2007).

3. Carvalho-Santos, Z., Azimzadeh, J., Pereira-Leal, J. B., Bettencourt-Dias, M. Evolution: Tracing the origins of centrioles, cilia, and flagella. Journal of Cell Biology. 194 (2), 165-175 (2011).

4. Randell, S. H., Boucher, R. C., University of North Carolina Virtual Lung, G. Effective mucus clearance 
is essential for respiratory health. American Journal of Respiratory Cell and Molecular Biology. 35 (1), 20-28 (2006).

5. Wanner, A., Salathe, M., O'Riordan, T. G. Mucociliary clearance in the airways. American Journal of Respiratory and Critical Care Medicine. 154 (6 Pt 1), 1868-1902 (1996).

6. Li, Y. et al. Global genetic analysis in mice unveils central role for cilia in congenital heart disease. Nature. $\mathbf{5 2 1}$ (7553), 520-524 (2015).

7. Zahid, M. et al. Airway ciliary dysfunction and respiratory symptoms in patients with transposition of the great arteries. PLoS One. 13 (2), e0191605 (2018).

8. Stewart, E. et al. Airway ciliary dysfunction: Association with adverse postoperative outcomes in nonheterotaxy congenital heart disease patients. Journal of Thoracic and Cardiovascular Surgery. 155 (2), 755-763 e757 (2018).

9. Garrod, A. S. et al. Airway ciliary dysfunction and sinopulmonary symptoms in patients with congenital heart disease. Annals of the American Thoracic Society. 11 (9), 1426-1432 (2014).

10. Harden, B. et al. Increased postoperative respiratory complications in heterotaxy congenital heart disease patients with respiratory ciliary dysfunction. Journal of Thoracic and Cardiovascular Surgery. 147 (4), 1291-1298 e1292 (2014).

11. Leigh, M. W. et al. Clinical and genetic aspects of primary ciliary dyskinesia/Kartagener syndrome. Genetics in Medicine. 11 (7), 473-487 (2009).
12. Donaldson, S. H. et al. Effect of ivacaftor on mucociliary clearance and clinical outcomes in cystic fibrosis patients with G551D-CFTR. JCI Insight. 3 (24) (2018).

13. Donaldson, S. H., Corcoran, T. E., Laube, B. L., Bennett, W. D. Mucociliary clearance as an outcome measure for cystic fibrosis clinical research. Proceedings of the American Thoracic Society. 4 (4), 399-405 (2007).

14. Ledowski, T., Hilmi, S., Paech, M. J. Bronchial mucus transport velocity in patients receiving anaesthesia with propofol and morphine or propofol and remifentanil. Anaesthesia. 61 (8), 747-751 (2006).

15. Donnelley, M., Morgan, K. S., Siu, K. K., Parsons, D. W. Dry deposition of pollutant and marker particles onto live mouse airway surfaces enhances monitoring of individual particle mucociliary transit behaviour. Journal of Synchrotron Radiation. 19 (Pt 4), 551-558 (2012).

16. Christopher, A. B. et al. The effects of temperature and anesthetic agents on ciliary function in murine respiratory epithelia. Frontiers in Pediatrics. 2111 (2014).

17. Liu, L. et al. Method for quantitative study of airway functional microanatomy using micro-optical coherence tomography. PLoS One. 8 (1), e54473 (2013).

18. Lam, H. C. et al. Histone deacetylase 6-mediated selective autophagy regulates COPD-associated cilia dysfunction. Journal of Clinical Investigation. 123 (12), $5212-5230$ (2013).

19. Bhashyam, A. R. et al. A pilot study to examine the effect of chronic treatment with immunosuppressive drugs on mucociliary clearance in a vagotomized murine model. PLoS One. 7 (9), e45312 (2012).

20. Ortiz, J. L. et al. Evaluation of Mucociliary Clearance by Three Dimension Micro-CT-SPECT in Guinea Pig: Role 
of Bitter Taste Agonists. PLoS One. 11 (10), e0164399 (2016).

21. Schindelin, J. et al. Fiji: an open-source platform for biological-image analysis. Nature Methods. 9 (7), 676-682 (2012).

22. Solomon, G. M. et al. Assessment of ciliary phenotype in primary ciliary dyskinesia by micro-optical coherence tomography. JCI Insight. 2 (5), e91702 (2017).

23. Nakhleh, N. et al. High prevalence of respiratory ciliary dysfunction in congenital heart disease patients with heterotaxy. Circulation. 125 (18), 2232-2242 (2012).

24. Whaley, S. L., Renken, S., Muggenburg, B. A., Wolff, R. K. Technique for aerosol deposition restricted to the nose in beagle dogs. Journal of Toxicology and Environmental Health. 23 (4), 519-525 (1988).

25. Foster, W. M., Walters, D. M., Longphre, M., Macri, K., Miller, L. M. Methodology for the measurement of mucociliary function in the mouse by scintigraphy. Journal of Applied Physiology (1985). 90 (3), 1111-1117 (2001).

26. Bhashyam, A. R. et al. Vagal control of mucociliary clearance in murine lungs: a study in a chronic preparation. Autonomic Neuroscience. 154 (1-2), 74-78 (2010).

27. Van Audenhaege, K. et al. Review of SPECT collimator selection, optimization, and fabrication for clinical and preclinical imaging. Medical Physics. 42 (8), 4796-4813 (2015). 